\section{Deep pockets}

\section{By Amy Donner, Senior Editor}

The B class of GPCRs is brimming with therapeutic targets, but thus far, development of small molecule drugs has been stymied. New studies reporting the first-ever crystal structures of members of the family could change that and open up new therapeutic real estate. ${ }^{1,2}$

GPCRs are versatile chemical sensors that coordinate cellular responses to a variety of extracellular stimuli and are the largest target class of FDA-approved drugs. ${ }^{3}$ Despite years of research and numerous approvals, predicting the relationship between ligands and GPCRs remains challenging because of a deficiency in molecular-level information about these interactions and the complexity of downstream biological effects.

Class B GPCRs are the second-largest class of the family. Members include receptors for peptide hormones such as glucagon (GCG), GCGlike peptide-1 (GLP-1), calcitonin gene-related peptide (CGRP), parathyroid hormone (PTH) and corticotropin-releasing factor 1 (CRF1).

"Class B GPCRs include many important drug targets, but there are no small molecule drugs for this class. The approved drugs are all peptide hormone ligands that need to be injected. Orally available, small molecule drugs are needed for these targets and these diseases," said Fiona Marshall, CSO at Heptares Therapeutics Ltd.

Now, a Heptares-led group has reported the structure of the transmembrane region of corticotropin-releasing factor receptor 1 (CRHR1; CRFR1) bound to the small molecule antagonist CP-376395 at $3 \AA$ resolution. ${ }^{1}$ A second group, led by Raymond Stevens and Ming-Wei Wang, solved the structure of the 7-transmembrane helical domain of GCG receptor (GCGR) at $3.4 \AA$ resolution. ${ }^{2}$

Stevens is a professor of molecular biology and chemistry at The Scripps Research Institute, and Wang is director at The National Center for Drug Screening and a professor of pharmacology at the Shanghai Institute of Materia Medica, Chinese Academy of Sciences.

The overall fold of the two structures resembles other published GPCR structures but with a key difference-CRHR1 and GCGR both feature a much larger cavity than class A GPCRs that faces the extracellular side of the membrane, presumably containing the peptidebinding site (see Figure 1, "Going deep").

In the cocrystal structure, the CRHR1 antagonist bound deep within this cavity. Indeed, the binding was so deep that the molecule was near the intracellular face of the plasma membrane.

According to Marshall, this is the biggest surprise revealed by the current structures. "The binding site is much lower down, approximately $15 \AA$ away from where typical GPCR drugs bind. This was entirely unexpected and provides a new area for scientists to focus on," she said.
Like class A GPCRs, the new structures feature a comparable arrangement of seven transmembrane helices and a similar alignment of portions of the receptors located on the intracellular side of the membrane.

Results of both studies were published in Nature. The team reporting the GCGR structure also included researchers from the Amsterdam Institute for Molecules, Medicines and Systems at the Free University Amsterdam, Stanford University and Novo Nordisk A/S.

On Wednesday, C4X Discovery Ltd. announced that it had solved the various $3 \mathrm{D}$ conformations of the antagonist reagent used in the CRHR1 study with its NMR-based technology.

\section{Building better models}

To aid its quest for small molecule drugs, the industry now will be able to build better models of class B GPCRs based on these structures and use these models, including the unexpectedly deep binding pocket, to inform structure-based design and virtual screening efforts.

"Models for class B receptors based on class A structures have not helped drug discovery efforts; nor have de novo models based solely on amino acid sequence. Before these structures, there was no evidence that the antagonist-binding pocket would be located where it is," said Marshall.

The sequence of amino acids is similar across GPCRs of the secretin family - the subset of class B receptors that contains CRHR1 and GCGR. Thus, the rest of the family should be amenable to modeling using the two new structures.

Both GPCRs are in an inactive conformation, and it remains to be determined what a bound agonist or an active conformation of class $\mathrm{B}$ receptors will look like.

Despite these challenges, Heptares plans to develop agonists of GLP-1 receptor (GLP-1R; GLP1R). The company hopes to take advantage of the new information emerging from these class B structures and combine it with prior information about the class A receptors to guide a virtual discovery effort.

Because the company is pursuing GLP-1R agonists, Marshall said that the goal will be to build a model of the active conformation of the receptor. To do so, the company will use structural information available from class A receptors in an active conformation in combination with the new class B structures.

Because of the similarity between class A and B structures in the portion of the receptor that interacts with downstream signaling components on
"Models for class B receptors based on class $A$ structures have not helped drug discovery efforts; nor have de novo models based solely on amino acid sequence. Before these structures, there was no evidence that the antagonistbinding pocket would be located where it is."

-Fiona Marshall, Heptares Therapeutics Ltd. conformations of these portions of the receptors will be comparable. 


\section{ANALYSIS}

\section{COVER STORY}

Figure 1. Going deep. GPCRs are high-priority targets for many indications including type 2 diabetes, depression, anxiety and osteoporosis. Hollenstein et al. have reported the first structure of an antagonist-bound class B GPCR and have shown that the antagonist-binding site in this class of receptors is much deeper than ligandbinding sites reported for class A. ${ }^{1} \mathrm{~A}$ second structure of a class B GPCR from Siu et al. extends the presence of a deep pocket to a second receptor, although there is no bound small molecule. ${ }^{2}$

(I) The relative location of the ligandbinding sites between class $A$ and class B GPCRs is shown in the overlay.

I

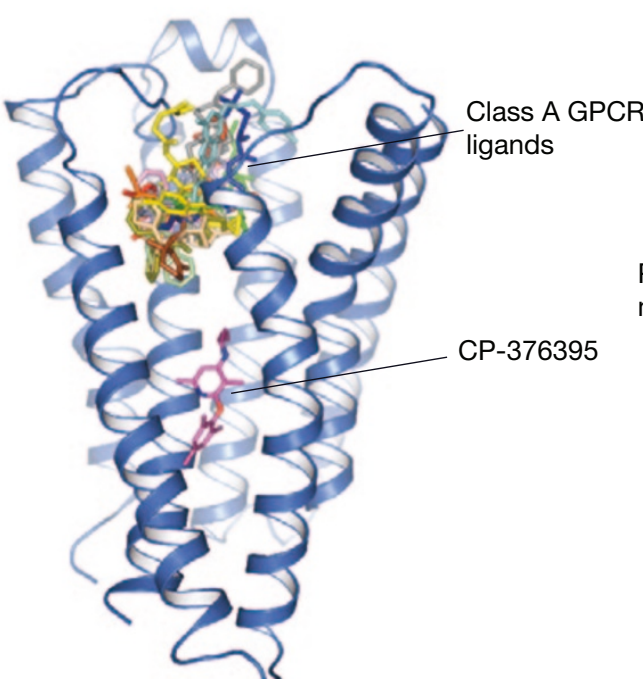

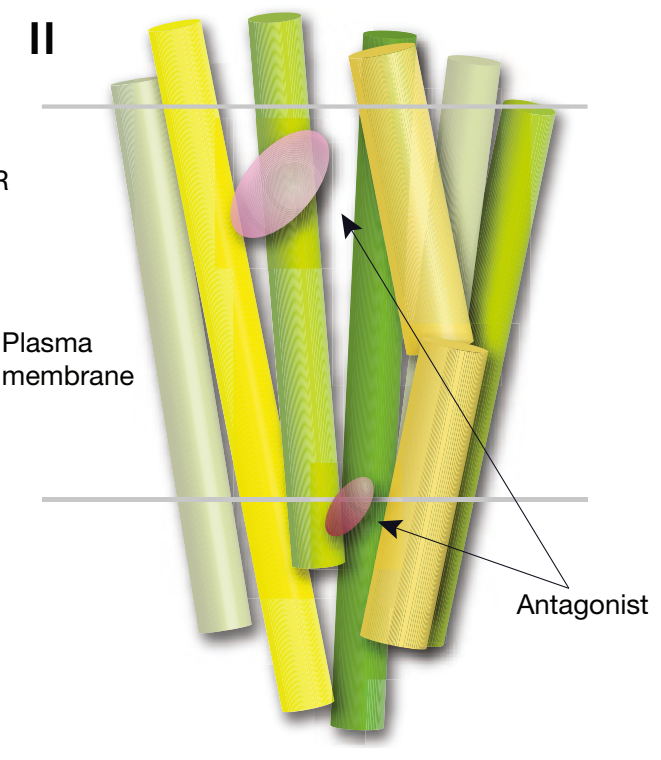

The class B binding site is occupied by a corticotropin-releasing factor receptor 1 (CRHR1; CRFR1) antagonist, and the location of an assortment of bound class A ligands, revealed in previously published structures, is indicated.

(II) Schematic representation of (I) showing the transmembrane helices embedded in the plasma membrane, with a deep pocket for the class B antagonist (red) near the intracellular plasma membrane interface and the shallow class A ligand-binding site (pink) near the extracellular interface.

There are more than 20 companies developing therapeutics that target class B GPCRs, but few of these programs involve an orally available small molecule. Neurocrine Biosciences Inc. and GlaxoSmithKline plc have a small molecule CRHR1 antagonist in Phase II testing for various neurological indications.

TransTech Pharma Inc. has a small molecule GLP-1R agonist in Phase II testing to treat diabetes. Addex Therapeutics Ltd. has a small molecule targeting GLP-1R in preclinical development for diabetes.

Heptares has filed for a patent on the CRHR1 structure. The patent and licensing status of the GCGR structure is unavailable. Stevens could not be reached for comment.

Donner, A. SciBX 6(32); doi:10.1038/scibx.2013.846

Published online Aug. 22, 2013

\section{REFERENCES}

1. Hollenstein, K. et al. Nature; published online July 17, 2013; doi:10.1038/nature12357

Contact: Fiona H. Marshall, Heptares Therapeutics Ltd., Welwyn Garden City, U.K.

e-mail: fiona.marshall@heptares.com
2. Siu, F.Y. et al. Nature; published online July 17, 2013; doi:10.1038/nature12393

Contact: Raymond C. Stevens, The Scripps Research Institute, La Jolla, Calif. e-mail: stevens@scripps.edu

Contact: Ming-Wei Wang, Shanghai Institute of Materia Medica, Chinese Academy of Sciences, Shanghai, China e-mail: wangmw@mail.shcnc.ac.cn

3. Granier, S. \& Kobilka, B. Nat. Chem. Biol. 8, 670-673 (2012)

COMPANIES AND INSTITUTIONS MENTIONED

Addex Therapeutics Ltd. (SIX:ADXN), Geneva, Switzerland C4X Discovery Ltd., Manchester, U.K.

Chinese Academy of Sciences, Beijing, China

Free University Amsterdam, Amsterdam, the Netherlands GlaxoSmithKline plc (LSE:GSK; NYSE:GSK), London, U.K. Heptares Therapeutics Ltd., Welwyn Garden City, U.K. The National Center for Drug Screening, Shanghai, China Neurocrine Biosciences Inc. (NASDAQ:NBIX), San Diego, Calif. Novo Nordisk A/S (CSE:NVO; NYSE:NVO), Bagsvaerd, Denmark The Scripps Research Institute, La Jolla, Calif. Shanghai Institute of Materia Medica, Shanghai, China Stanford University, Stanford, Calif. TransTech Pharma Inc., High Point, N.C. 